Constr. Approx. (1991) 7: 209-220

CONSTRUCTIVE APPROXIMATION

\title{
On Computational Aspects of Simplicial Splines
}

\author{
M. Neamtu and C. R. Traas
}

\begin{abstract}
Some new results on multivariate simplex B-splines and their practical application are presented. New recurrence relations are derived based on [2] and [15]. Remarks on boundary conditions are given and an example of an application of bivariate quadratic simplex splines is presented. The application concerns the approximation of a surface which is constrained by a differential equation.
\end{abstract}

\section{Introduction}

The multivariate simplex B-splines possess a number of attractive properties which make them suitable, in principle, for describing geometrical objects: they have local support, piecewise polynomial character, and global smoothness. Efficient methods for their numerical treatment have been developed.

In this article we present some recent results obtained in connection with our effort toward a practical exploitation of these splines. First, in Section 2, some new recurrence relations are derived based on the results in [2] and [15]. Next, in Section 3, some remarks are made about B-splines as finite-element functions, in particular about satisfying boundary conditions. Finally, an example of an application of B-splines is presented for the case of a surface constrained by a differential equation (the finite-element method). In relation to the application we restrict ourselves to bivariate quadratic B-splines to keep the programming complexity low.

\section{Recurrence Relations for Computing with B-Splines}

In this section we derive some new recurrence relations for B-splines. They are based on some recent results, in particular on the recurrence relations derived in [2] and the notion of multivariate divided difference defined in [15] and [16].

Let $i$ be both a multi-index $\left(i_{1}, \ldots, i_{s}\right)$ and a set $\left\{i_{1}, \ldots, i_{s}\right\}$, where $i_{j} \in\{0,1,2, \ldots\}$, $j=1, \ldots, s, s \geq 1$. $S$ denotes the set of all $s$-tuples $\mathbf{i}=\left(i_{1}, \ldots, i_{s}\right)$, such that $i_{j} \in N:=\{0, \ldots, n\}, n \geq s$, and $i_{j} \neq i_{k}$ for $j \neq k ; j, k=1, \ldots, s$. Moreover, $\mathbf{x}^{i}$ denotes an s-tuple $\left(\mathbf{x}^{i_{1}}, \ldots, \mathbf{x}^{i_{\boldsymbol{z}}}\right), \mathbf{x}^{i_{j}} \in \mathbf{R}^{\mathbf{s}}$.

Date received: April 26, 1989. Date revised: September 20, 1989. Communicated by Charles Micchelli. AMS classification: $41 \mathrm{A15}, 41 \mathrm{A63}, 65 \mathrm{D07}$.

Key words and phrases: Multivariate B-splines, Simplex splines, Cone splines, Recurrence relations, Multivariate divided differences. 
Let $\mathbf{x}^{0}, \ldots, \mathbf{x}^{n} \in \mathbf{R}^{s}$ be knots in general position [9] and let $f\left(\mathbf{x}^{\mathbf{j}}\right)$ be a real-valued function of $s$ vector variables $x^{i_{1}}, \ldots, \mathbf{x}^{i_{s}}$. Then the multivariate divided difference of $f$ is defined by [15], [16]

$$
\left[\mathbf{x}^{0}, \ldots, \mathbf{x}^{n}\right] f:=\sum_{\mathbf{i} \in S} \frac{f\left(\mathbf{x}^{\mathrm{i}}\right)}{\prod_{p \in N \backslash \mathbf{i}} d\left(\mathbf{x}^{\mathbf{i}}, \mathbf{x}^{p}\right)},
$$

where

$$
d\left(\mathbf{x}^{\mathbf{i}}, \mathbf{x}^{p}\right):=d\left(\mathbf{x}^{\mathbf{i}}, \mathbf{x}^{p}, 1\right)
$$

and

$$
d\left(\mathbf{x}^{\mathbf{i}}, \mathbf{x}^{p}, c\right):=\operatorname{det}\left[\begin{array}{cccc}
\mathbf{x}^{i_{1}} & \cdots & \mathbf{x}^{i_{s}} & \mathbf{x}^{p} \\
1 & \cdots & 1 & c
\end{array}\right], \quad c \in \mathbf{R} .
$$

Instead of (2.1) we also make use of the equivalent notation

$$
\left[\mathbf{x}^{0}, \ldots, \mathbf{x}^{n}\right] f=:\left[\mathbf{x}^{0}, \ldots, \mathbf{x}^{n}\right] f(\cdot)=:\left[\mathbf{x}^{0}, \ldots, \mathbf{x}^{n}\right]_{\mathbf{i}} f\left(\mathbf{x}^{\mathbf{i}}\right) .
$$

Let the operators $\varphi, \mu, \mu / \varphi$ be defined for $\mathbf{x}=\left(x_{i}, \ldots, x_{s}\right) \in \mathbf{R}^{s}$ and $\mathbf{i}=\left(i_{i}, \ldots, i_{s}\right) \in S$ by

$$
\varphi \mathrm{x}=x_{1}, \quad \mu \mathrm{x}=\left(x_{2}, \ldots, x_{s}\right), \quad \frac{\mu}{\varphi} \mathrm{x}=\left(\frac{x_{2}}{x_{1}}, \ldots, \frac{x_{s}}{x_{1}}\right) \text { for } x_{1} \neq 0
$$

$$
\varphi \mathbf{i}=i_{1}, \quad \mu \mathbf{i}=\left(i_{2}, \ldots, i_{s}\right) .
$$

Let $T^{(k)}$ be a multivariate function defined for $k>0$ as

$$
T^{(k)}\left(\mathbf{x}^{\mathbf{i}}, \mathbf{x}\right):=T^{(0)}\left(\mathbf{x}^{\mathbf{i}}, \mathbf{x}\right) \cdot d^{k}\left(\mathbf{x}^{\mathbf{i}}, \mathbf{x}\right)
$$

$T^{(0)}$ is defined recursively as

$$
\begin{aligned}
& T^{(0)}(a, b):=\eta(a-b), \quad a, b \in \mathbf{R}, \\
& T^{(0)}\left(\mathbf{x}^{\mathbf{1}}, \mathbf{x}\right):=-T^{(0)}\left(\varphi \mathbf{x}^{i}, \varphi \mathbf{x}\right) T^{(0)}\left(\left\{\frac{\mu}{\varphi}\left(\mathbf{x}^{k}-\mathbf{x}^{i}\right)\right\}_{k \in \mathcal{X} \backslash i}, \frac{\mu}{\varphi}\left(\mathbf{x}-\mathbf{x}^{i}\right)\right) .
\end{aligned}
$$

Here

$$
\eta(t):=\left\{\begin{array}{ll}
1, & t \geq 0, \\
0, & t<0,
\end{array} \quad i:=\varphi \mathbf{i}\right.
$$

It has been shown in [16] (see also [15]) that this appropriate definition of $T^{(k)}$ gives rise to the following relation between multivariate B-splines and multivariate divided differences:

$$
M(\mathbf{x} \mid X)=\frac{n !}{(n-s) !}\left[\mathbf{x}^{0}, \ldots, \mathbf{x}^{n}\right] T^{(n-s)}(\cdot, \mathbf{x}),
$$

where $M(\mathbf{x} \mid X)$ is the multivariate B-spline with knots $X:=\left\{\mathbf{x}^{0}, \ldots, \mathbf{x}^{n}\right\}[8]$.

This relation is valid whenever the knots $X$ are in general position and such that the function $T^{(0)}$ is, by (2.4), well defined (i.e., no division by zero occurs). In analogy to the univariate case the function $T$ can be considered as another generalization of the truncated power function, different from the one given in [3].

We employ this relation later. To this end we point out that if $A(s \times s)$ is any 
nonsingular matrix with $\operatorname{det}(A)=1$ and $\overline{\mathbf{x}}=A \mathbf{x}$ it follows from the definition of the multivariate B-spline that

$$
M(\mathbf{x} \mid X)=M(\overline{\mathbf{x}} \mid \bar{X})
$$

where $\bar{X}=A X=\left\{A \mathbf{x}^{0}, \ldots, A \mathbf{x}^{n}\right\}$. Thus, we can write

$$
M(\mathbf{x} \mid X)=\frac{n !}{(n-s) !}\left[\overline{\mathbf{x}}^{0}, \ldots, \overline{\mathbf{x}}^{n}\right] T^{(n-s)}(\cdot, \overline{\mathbf{x}}) .
$$

Relation (2.7) holds whenever matrix $A$ is chosen such that the right-hand side of (2.7) is defined. Similarly, if $\mathbf{z}^{1}, \ldots, \mathbf{z}^{p} \in \mathbf{R}^{s}, p \in\{1,2, \ldots\}$, then

$$
D_{\mathbf{z}^{1}, \ldots, z^{p}} M(\mathbf{x} \mid X):=D_{\mathbf{z}^{1}} \cdots D_{\mathbf{z}^{p}} M(\mathbf{x} \mid X)=D_{\overline{\mathbf{z}}^{1} 1 \ldots, \overline{\mathbf{z}}^{p}} M(\overline{\mathbf{x}} \mid \bar{X}),
$$

whenever both sides of (2.8) are defined.

Remark 2.1. From now on we suppress the upper bars from the notation. Thus instead of expressions of type (2.7), type (2.5) will be used. However, care must be taken as sometimes expressions of type (2.5) are not defined, while (2.7) holds. Therefore, we consider (2.5) as an expression which says that the equality holds iff an appropriate linear transform $\mathbf{x} \rightarrow A \mathbf{x}$ with $\operatorname{det}(A)=1$ is taken rather than $\mathbf{x} \rightarrow \mathbf{x}$.

Finally, we recall that whenever

$$
0 \notin\left[X^{i}\right]:=\left[\mathrm{x}^{i}-\mathrm{x}^{0}, \ldots, \mathrm{x}^{i}-\mathrm{x}^{i-1}, \mathrm{x}^{i+1}-\mathrm{x}^{i}, \ldots, \mathrm{x}^{n}-\mathrm{x}^{i}\right], \quad i=0, \ldots, n,
$$

then $[3]$

$$
M(\mathbf{x} \mid X)=n ! \sum_{i=0}^{n}(-1)^{i} C\left(\mathbf{x}-\mathbf{x}^{i} \mid X^{i}\right)
$$

where $C\left(\cdot \mid X^{i}\right)$ denotes the cone spline (truncated power function) with knots $X^{i}[3]$.

\subsection{Recurrence Relation for B-Splines with Respect to Dimension}

Recently a new recurrence relation for multivariate B-splines was derived by Cohen et al. [2]. For completeness and our later purposes we summarize the main results in [2] in a slightly modified form.

Assume that $\varphi \mathbf{x}^{1}, \ldots, \varphi \mathbf{x}^{n}>0$. Then

$$
C\left(\mathbf{x} \mid \mathbf{x}^{1}, \ldots, \mathbf{x}^{n}\right)=\frac{(\varphi \mathbf{x})_{+}^{n-s} M\left((\mu / \varphi) \mathbf{x} \mid(\mu / \varphi) \mathbf{x}^{1}, \ldots,(\mu / \varphi) \mathbf{x}^{n}\right)}{(n-1) ! \prod_{i=1}^{n} \varphi \mathbf{x}^{i}}
$$

Moreover, for

$$
\begin{gathered}
\varphi \mathbf{x}^{0}<\varphi \mathbf{x}^{1}<\cdots<\varphi \mathbf{x}^{n} \quad \text { and } \varphi \mathbf{x} \neq \varphi \mathbf{x}^{i} \quad(i=0, \ldots, n), \\
M(\mathbf{x} \mid X)=(-1)^{n} n\left[\varphi \mathbf{x}^{0}, \ldots, \varphi \mathbf{x}^{n}\right]_{i}\left(\varphi \mathbf{x}-\varphi \mathbf{x}^{i}\right)_{+}^{n-s} M_{i}
\end{gathered}
$$

where

$$
M_{i}:=M\left(\frac{\mu}{\varphi}\left(\mathbf{x}-\mathbf{x}^{i}\right) \mid\left\{\frac{\mu}{\varphi}\left(\mathbf{x}^{p}-\mathbf{x}^{i}\right)\right\}_{p \in N \backslash i}\right)
$$


Here $\left[\varphi \mathbf{x}^{0}, \ldots, \varphi \mathbf{x}^{n}\right]_{i} f\left(\varphi \mathbf{x}^{i}\right)$ stands for the univariate divided difference with nodes $\varphi \mathbf{x}^{0}, \ldots, \varphi \mathbf{x}^{n}$.

Expression (2.12) is to be considered in the sense of Remark 2.1. Thus the knots on the right-hand side of (2.12) can be viewed to be transformed by a matrix $A$ with $\operatorname{det}(A)=1$. As mentioned in [15], relation (2.12) also follows directly from (2.5) by exploiting the properties of the multivariate divided difference functional.

From (2.12) we have the following recurrence relation [2]. $M(\mathbf{x} \mid X)$ can be computed recursively as

$$
\begin{array}{r}
M_{k, s}=\left[\varphi \mathbf{x}^{k}, \ldots, \varphi \mathbf{x}^{k+s}\right]_{i} \eta\left(\varphi \mathbf{x}-\varphi \mathbf{x}^{i}\right) M\left(\frac{\mu}{\varphi}\left(\mathbf{x}-\mathbf{x}^{i}\right) \mid\left\{\frac{\mu}{\varphi}\left(\mathbf{x}^{p}-\mathbf{x}^{i}\right)\right\}_{p \in N \backslash i}\right), \\
k=0, \ldots, n-s,
\end{array}
$$

$$
\begin{gathered}
M_{k, j}=\frac{\left(\varphi \mathbf{x}-\varphi \mathbf{x}^{k}\right) M_{k, j-1}+\left(\varphi \mathbf{x}^{k+j}-\varphi \mathbf{x}\right) M_{k+1, j-1}}{\varphi \mathbf{x}^{k+j}-\varphi \mathbf{x}^{k}}, \quad \\
\quad j=s+1, \ldots, n, \quad k=0, \ldots, n-j, \\
M(\mathbf{x} \mid X)=n M_{0, n}
\end{gathered}
$$

Note that (2.12) is an analogy to a well-known univariate result (cf. (2.19)). It follows from (2.13)-(2.15) that the multivariate B-spline is expressed in terms of its lower-dimensional B-splines. Thus, (2.13)-(2.15) is referred to as the recurrence relation with respect to dimension, which is in contrast with the recurrence relation with respect to order [15].

\subsection{Evaluation of Directional Derivatives of $B$-Splines}

Before dealing with the evaluation of the directional derivatives of a B-spline we consider the following problem. Let $\mathbf{v}^{j} \in \mathbf{R}^{s}$ and $c_{j} \in \mathbf{R}(j=1, \ldots, n-s)$.

We are interested in an efficient evaluation of the expression

$$
\left[\mathbf{x}^{0}, \ldots, \mathbf{x}^{n}\right]_{i} \prod_{j=1}^{n-s} d\left(\mathbf{x}^{\mathbf{1}}, \mathbf{v}^{j}, c_{j}\right) f_{i},
$$

where $f_{\mathbf{i}} \in \mathbf{R}$ and $\mathbf{i} \in S$, provided that (2.16) is defined. We first give a recurrence relation for (2.16).

Lemma 2.1.

$$
\begin{aligned}
{\left[\mathbf{x}^{0}, \ldots,\right.} & \left.\mathbf{x}^{n}\right]_{i} \prod_{j=1}^{n-s} d\left(\mathbf{x}^{\mathbf{i}}, \mathbf{v}^{j}, c_{j}\right) f_{\mathbf{i}} \\
= & (-1)^{n+1}\left[\varphi \mathbf{x}^{0}, \ldots, \varphi \mathbf{x}^{n}\right]_{i}\left(\prod_{j=1}^{n-s} \varphi\left(\mathbf{v}^{j}-c_{j} \mathbf{x}^{i}\right)\left[\left\{\frac{\mu}{\varphi}\left(\mathbf{x}^{p}-\mathbf{x}^{i}\right)\right\}_{p \in N \backslash i}\right]_{i \backslash i}\right. \\
& \left.\times \prod_{j=1}^{n-s} d\left(\left\{\frac{\mu}{\varphi}\left(\mathbf{x}^{p}-\mathbf{x}^{i}\right)\right\}_{p \in i \backslash i}, \frac{\mu}{\varphi}\left(\mathbf{v}^{j}-c_{j} \mathbf{x}^{i}\right), 1\right) f_{i}\right),
\end{aligned}
$$

where $i:=\varphi \mathbf{i}$. The equality holds if both sides of (2.17) are defined. 
Proof. The proof follows directly from the definition of the multivariate divided difference (2.1) (see [16] for details).

Lemma 2.1 gives a recurrence relation for expressions of type (2.16). Thus, (2.16) can be expressed in terms of lower-dimensional expressions of the same type. Hence, relation (2.17) can be viewed as a recurrence relation for expression (2.16) with respect to dimension. In fact, notice that (2.12) is a special case of (2.17).

We can see from (2.17) that evaluation of (2.16) can be accomplished by a successive evaluation of univariate divided differences. Hence, in order to employ (2.17) we need to calculate expressions of the type

$$
E:=\left[x^{0}, \ldots, x^{n}\right]_{i} \prod_{j=1}^{n-1}\left(v^{j}-c_{j} x^{i}\right) g_{i}
$$

where $x^{i}:=\varphi \mathbf{x}^{i}, i:=\varphi \mathbf{i}, v^{j}:=\varphi \mathbf{v}^{j}$, and $g_{i} \in \mathbf{R}$.

This expression can be considered as a generalization of the well-known expression for the univariate B-splines

$$
(-1)^{1} n\left[x^{0}, \ldots, x^{n}\right](x-\cdot)_{+}^{n-1} .
$$

Note, that expressions of the type $\left[x^{0}, \ldots, x^{n}\right]_{i} \prod_{j=1}^{n-s}\left(v^{j}-c_{j} x^{i}\right) g_{i}$ are special cases of $(2.18)$ with $c_{j}=0, v_{j}=1$ for $j=n-s+1, \ldots, n-1$.

Lemma 2.2. E can be evaluated recursively as

$$
\begin{aligned}
E_{k, 1} & =\left[x^{k}, x^{k+1}\right]_{i} g_{i}, \quad k=0, \ldots, n-1, \\
E_{k, j} & =\frac{\left(v^{j-i}-c_{j-1} x^{k}\right) E_{k, j-1}+\left(c_{j-1} x^{k+j}-v^{j-1}\right) E_{k+1, j-1}}{x^{k+j}-x^{k}} \quad j=2, \ldots, n, \quad k=0, \ldots, n-j, \\
E & =E_{0, n} .
\end{aligned}
$$

Proof. The proof is similar to the well-known one for the univariate B-splines (2.19). It requires a standard use of the Leibniz rule for divided differences.

Remark 2.2. The recurrence relation given in Lemma 2.2 can be considered as a generalization of the well-known recurrence relation for the univariate B-splines as well as of (2.13)-(2.15). The computational complexity of this recurrence relation is the same, however, as the only difference is that at each level $j$, different values of $\mathbf{v}$ are used.

On the other hand, if $v^{j}=1, c_{j}=0(j=1, \ldots, n-1)$, then (2.20)-(2.22) reduce to the familiar divided difference scheme. Thus, the recurrence relations for the univariate B-splines, divided differences, and multivariate B-splines (2.13)-(2.15) are special cases of (2.20)-(2.22).

It turns out that the general recurrence relations given in Lemmas 2.1 and 2.2 are also useful for computing the directional derivatives of multivariate B-splines. 
Theorem 2.1. Let $\mathbf{x}^{0}, \ldots, \mathbf{x}^{n}$ be in general position and let $\mathbf{z}^{1}, \ldots, \mathbf{z}^{p} \in \mathbf{R}^{s}, 1 \leq p \leq$ $n-s$, then

$$
D_{\mathbf{z}^{1}}, \ldots, \mathbf{z}^{p} M(\mathbf{x} \mid X)=\frac{n !}{(n-s-p) !}\left[\mathbf{x}^{0}, \ldots, \mathbf{x}^{n}\right] T^{n-s-p}(\cdot, \mathbf{x}) \prod_{i=1}^{p} d\left(\cdot, \mathbf{z}^{i}, 0\right) .
$$

The equality is to be taken in the sense of Remark 2.1 .

Proof. The proof follows from (2.5) and the definition of $T(2.3),(2.4)$.

Note, that the expression on the right-hand side of (2.23) is a special case of (2.16). Hence, employing Lemmas 2.1 and 2.2 we can evaluate the directional derivatives of B-splines.

\subsection{Evaluation of Inner Products of B-Splines}

Let us first recall that if $C\left(\mathbf{x} \mid \mathbf{x}^{1}, \ldots, \mathbf{x}^{m}\right), C\left(\mathbf{x} \mid \mathbf{y}^{1}, \ldots, \mathbf{y}^{n}\right)$ are two cone splines with $0 \notin[X]:=\left[\mathbf{x}^{1}, \ldots, \mathbf{x}^{m}\right]$ and $0 \notin[Y]:=\left[y^{1}, \ldots, y^{n}\right]$, then their convolution gives another cone spline [5], namely

$$
\begin{aligned}
\int_{\mathbf{R}^{s}} C\left(\mathbf{y}-\mathbf{x} \mid \mathbf{x}^{1}, \ldots, \mathbf{x}^{m}\right) C\left(\mathbf{x} \mid \mathbf{y}^{1}, \ldots, \mathbf{y}^{n}\right) d \mathbf{x} & =C\left(\mathbf{y} \mid \mathbf{x}^{1}, \ldots, \mathbf{x}^{m}, \mathbf{y}^{1}, \ldots, \mathbf{y}^{n}\right) \\
& =C(\mathbf{y} \mid X, Y) .
\end{aligned}
$$

In particular

$$
\int_{\mathbf{R}^{s}} C(\mathbf{x} \mid X) C(\mathbf{x} \mid Y) d \mathbf{x}=C(0 \mid-X, Y)
$$

where $-X:=\left\{-\mathbf{x}^{1}, \ldots,-\mathbf{x}^{m}\right\}$. We also recall the notation for the inner products of B-splines:

$$
I(X \mid Y):=\int_{R^{s}} M(\mathbf{x} \mid X) M(\mathbf{x} \mid Y) d \mathbf{x} .
$$

Combining (2.25) with (2.9) and (2.10) gives the next theorem, which is to be understood in the sense of Remark 2.1 .

Theorem 2.2. Let $M=\{0, \ldots, m\}, N=\{0, \ldots, n\}$, and $m, n \geq s$. If the knots $X$ are pairwise distinct, as well as the knots of $Y$, then

$$
\begin{aligned}
I(X \mid Y)= & \frac{(-1)^{m} m ! n !}{(m+n-1) !}\left[\varphi \mathbf{x}^{0}, \ldots, \varphi \mathbf{x}^{m}\right]_{i}\left[\varphi \mathbf{y}^{0}, \ldots, \varphi \mathbf{y}^{n}\right]_{j} \\
& \times\left(\varphi \mathbf{y}^{j}-\varphi \mathbf{x}^{i}\right)_{+}^{m+n-s} M_{i, j},
\end{aligned}
$$

where $M_{i, j}$ is an $(s-1)$-dimensional B-spline defined as

$$
M_{i, j}:=M\left(\frac{\mu}{\varphi}\left(y^{j}-x^{i}\right) \mid\left\{\frac{\mu}{\varphi}\left(x^{k}-x^{i}\right)\right\}_{k \in M \backslash i}\left\{\frac{\mu}{\varphi}\left(y^{p}-y^{j}\right)\right\}_{p \in N \backslash}\right) .
$$

We point out that relation (2.26) is almost identical with the univariate formula $\left[1\right.$, p. 132], except for the factor $M_{i, j}$. In analogy to the recurrence relation with respect to dimension (2.12), the inner product $I(X \mid Y)$ is expressed in terms of 
lower-dimensional B-splines, thus we call (2.26) a recurrence relation with respect to dimension.

Proof.

$$
\begin{aligned}
I(X \mid Y)= & \int_{\mathbf{R}^{s}} M(\mathbf{x} \mid X) M(\mathbf{x} \mid Y) d \mathbf{x} \\
= & m ! n ! \int_{\mathbf{R}^{s}}\left(\sum_{i=0}^{m}(-1)^{i} C\left(\mathbf{x}-\mathbf{x}^{i} \mid X^{i}\right)\right)\left(\sum_{j=0}^{n}(-1)^{j} C\left(\mathbf{x}-\mathbf{y}^{j} \mid Y^{j}\right)\right) d \mathbf{x} \\
= & m ! n ! \sum_{i=0}^{m} \sum_{j=0}^{n}(-1)^{i+j} \int_{\mathbf{R}^{s}} C\left(\mathbf{x}-\mathbf{x}^{i} \mid X^{i}\right) C\left(\mathbf{x}-\mathbf{y}^{j} \mid Y^{j}\right) d \mathbf{x} \\
= & m ! n ! \sum_{i=0}^{m} \sum_{j=0}^{n}(-1)^{i+j} C\left(\mathbf{y}^{j}-\mathbf{x}^{i} \mid X^{i},-Y^{j}\right) \\
= & \frac{m ! n !}{(m+n-1) !} \sum_{i=0}^{m} \sum_{j=0}^{n} \frac{(-1)^{i+j}}{\varphi\left(\mathbf{x}^{i}-\mathbf{x}^{0}\right) \cdots \varphi\left(\mathbf{x}^{i}-\mathbf{x}^{i-1}\right) \varphi\left(\mathbf{x}^{i+1}-\mathbf{x}^{i}\right) \cdots \varphi\left(\mathbf{x}^{m}-\mathbf{x}^{i}\right)} \\
& \times \frac{\left(\varphi \mathbf{y}^{j}-\varphi \mathbf{x}^{i}\right)_{+}^{m+n-s}}{\varphi\left(\mathbf{y}^{0}-\mathbf{y}^{j}\right) \cdots \varphi\left(\mathbf{y}^{j-1}-\mathbf{y}^{j}\right) \varphi\left(\mathbf{y}^{j}-\mathbf{y}^{j+1}\right) \cdots \varphi\left(\mathbf{y}^{j}-\mathbf{y}^{n}\right)} \\
& \times M\left(\frac{\mu}{\varphi}\left(\mathbf{y}^{j}-\mathbf{x}^{i}\right) \mid\left\{\frac{\mu}{\varphi}\left(\mathbf{x}^{k}-\mathbf{x}^{i}\right)\right\}_{k \in M M^{\prime}}\left\{\frac{\mu}{\varphi}\left(\mathbf{y}^{p}-\mathbf{y}^{j}\right)\right\}_{p \in N \backslash j}\right) \\
= & \frac{(-1)^{m} m ! n !}{(m+n-1) !}\left[\varphi \mathbf{x}^{0}, \ldots, \varphi \mathbf{x}^{m}\right]_{i}\left[\varphi \mathbf{y}^{0}, \ldots, \varphi \mathbf{y}^{n}\right],\left(\varphi \mathbf{y}^{j}-\varphi \mathbf{x}^{i}\right)_{+}^{m+n-s} M_{i, j} \quad \mathbf{m}
\end{aligned}
$$

Following the same argument for $I(X \mid Y)$ as in the univariate case [1], we can derive the next recurrence relation.

Denote

$$
I_{i, j}^{k, p}:=(-1)^{k}\left[x^{i}, \ldots, x^{i+k}\right]_{r}\left[y^{j}, \ldots, y^{j+p}\right]_{t}\left(y^{t}-x^{r}\right)_{+}^{k+p-s} M_{r, t},
$$

where $x^{i}:=\varphi \mathbf{x}^{i}$ and $y^{j}:=\varphi \mathbf{y}^{j}$. Then:

1. For $k+p=s, k, p>0$,

$$
I_{i, j}^{k, p}=(-1)^{k}\left[x^{i}, \ldots, x^{i+k}\right]_{\mathrm{r}}\left[y^{j}, \ldots, y^{j+p}\right]_{\mathrm{r}} \eta\left(y^{\ell}-x^{k}\right) M_{\mathrm{r}, t} .
$$

2. For $k+p>s, k, p>0$,

$$
I_{i, j}^{k, p}=I_{i+1, j}^{k, p}+\frac{\left(x^{i}-y^{j}\right) I_{i, j}^{k, p-1}+\left(y^{j+p}-x^{i}\right) I_{i, j+1}^{k, p}}{\left(y^{j+p}-y^{j}\right)}
$$

or

$$
I_{i, j}^{k, p}=\frac{\left(y^{j}-x^{i}\right) I_{i, j}^{k-1, p}+\left(x^{i+k}-y^{j}\right) I_{i+1, j}^{k, p}}{\left(x^{i+k}-x^{i}\right)}+I_{i, j+1}^{k, p}
$$

3.

$$
I(X \mid Y)=\frac{m ! n !}{(m+n-1) !} I_{0,0}^{m, n}
$$


Based on (2.29)-(2.32) we can devise an algorithm for the evaluation of inner products of B-splines.

More about the practical exploitation of the recurrence relations presented here and numerical experiments appear elsewhere [14].

\section{B-splines as Basis Functions in the Finite-Element Method}

In this section we consider spaces spanned by collections of B-splines in relation to a finite-element computation. The main ingredients needed for such an application are:

- general rules for constructing an approximation space;

-rules for constructing B-splines near boundaries such that linear combinations of them satisfy given conditions;

-an algorithm for computing inner products of B-splines.

The problem of constructing an approximation space has been described extensively in the literature (e.g., [4], [6], [7]-[10], [13]). The method used-starting with a "proper" triangulation with multiple knots of a domain $\Omega$ in $\mathbf{R}^{s}$ and then "pulling the knots apart"-is relatively well known nowadays and needs no repetition in this article.

Rules for bivariate quadratic B-splines near boundaries, in relation to boundary conditions, are described in [10] and [18]. In [10] it is stated that the boundary condition $u=0, \partial u / \partial n \neq 0$ can be satisfied by pulling, from every boundary point, one point along the boundary itself and the other point to the interior of $\Omega$, where the typifications of the vertices and of the pulled points (near the boundary) are exactly specified. It should be remarked that several alternative typifications exist which allow satisfaction of the above boundary condition. However, on the other hand, there exist typifications for which this boundary condition cannot be satisfied. In other words, typification of vertices on the boundary and of pulled knots should be done with prudence. An example of a boundary situation in which $u=0$, and where $\partial u / \partial n \neq 0$ cannot be satisfied everywhere is given in Fig. 3.1. In the point $P, u_{n}$ vanishes.

The large numbers $0,1,2$ are the typifications of the vertices $P, Q, R$ with respect

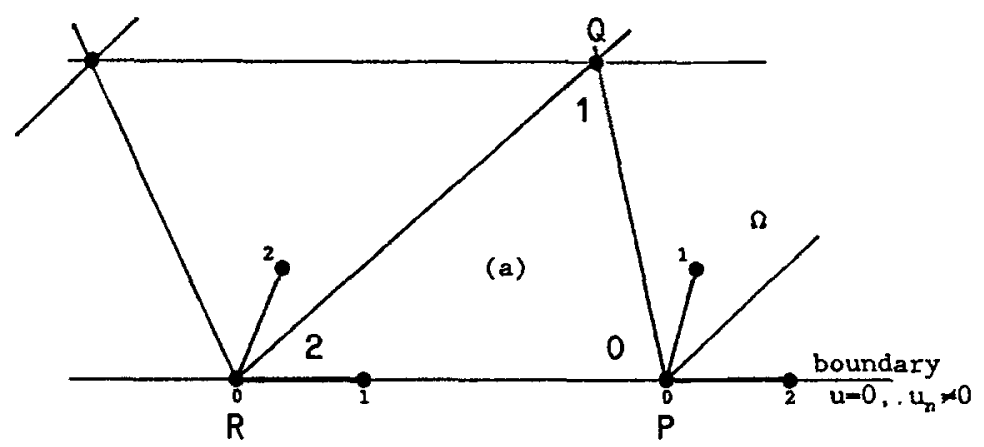

Fig. 3.1. $u_{n} \neq 0$ cannot be satisfied everywhere on the boundary. 


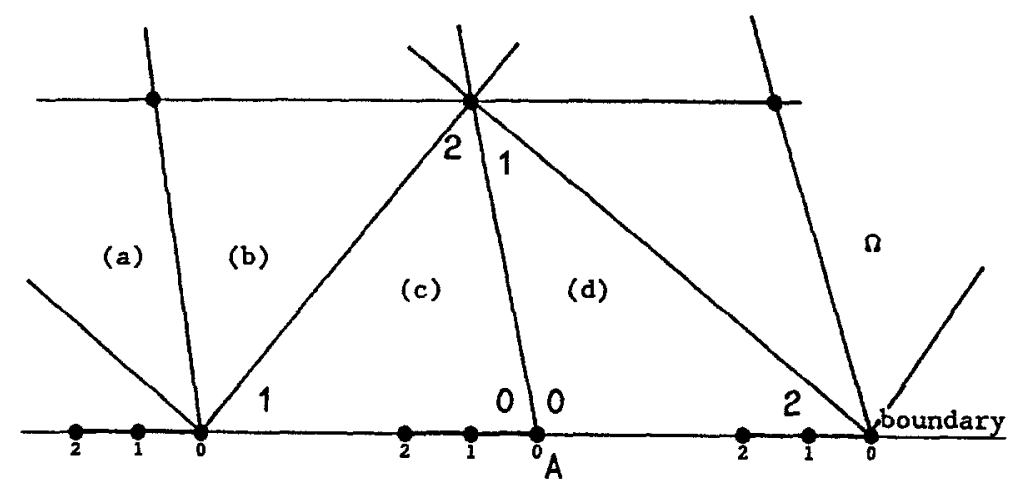

Fig. 3.2. The symmetry condition cannot be satisfied in $A_{0}$ or in $A_{2}$.

to triangle (a), and the small numbers $0,1,2$ are the typifications of the original knot and of the pulled ones, respectively. Special care should also be taken when intending to satisfy a symmetry condition $u \neq 0, \partial u / \partial n=0$ along a boundary [18]. Here the configuration of triangles, adjoining the boundary, should be such that the prescription $u_{n}=0$ can be satisfie d everywhere along this boundary. An example of a situation in which the symmetry condition cannot be satisfied everywhere is given in Fig. 3.2. The remedy is to revise the triangulation locally.

In [18] a cornerpoint of $\Omega$ is described in which two boundaries with different boundary conditions meet. In that publication the cornerpoint has been taken single, requiring an extension of $\Omega$ in order to allow the boundary conditions to be satisfied locally. It appears to be possible to assign multiplicity 2 to the cornerpoint, by careful local type assignment to vertices and to pulled points. Of course then no edge inside $\Omega$ may have this point as its origin, otherwise "nonsmoothness" would propagate into $\Omega$. An example of such a junction is given in Fig. 3.3.

In Section 4 we compute the shape of a thin plate which is subject to forces perpendicular to the plate, using quadratic B-splines. In relation to that application we have to evaluate inner products containing second-order derivatives, e.g.,

$$
\iint_{\Omega} \frac{\partial^{2} M(\mathbf{x} \mid X)}{\partial x^{2}} \frac{\partial^{2} M(\mathbf{x} \mid Y)}{\partial x^{2}} d x d y .
$$

Here $\Omega \subset \mathbf{R}^{2}$ is the region of integration (the plate), $X$ and $Y$ are knot sets

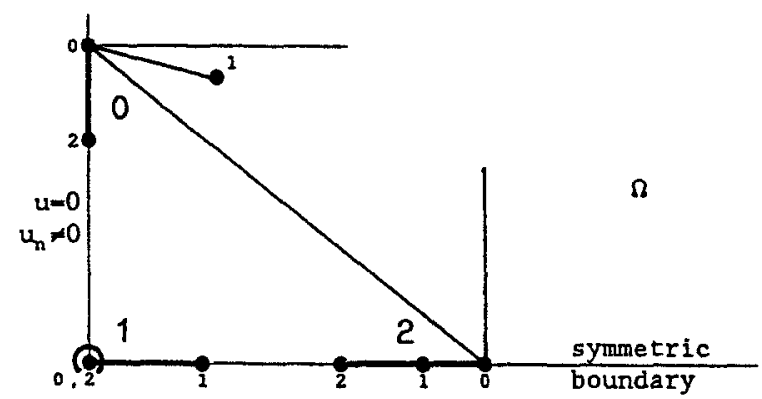

Fig. 3.3. Local typification, allowing junction of two boundaries. 
containing five knots each, and $\mathbf{x}=(x, y)$. A second-order derivative of a quadratic $B$-spline can be written as a linear combination of a number of piecewise constant B-splines. Using this fact, (3.1) can be written in term of inner products of the form

$$
I(X \mid Y)=\iint_{\mathbf{\Omega}} M(\mathbf{x} \mid X) M(\mathbf{x} \mid Y) d x d y
$$

in which $X$ and $Y$ are now knot sets containing three knots each. Since the B-splines occurring in (3.2) are of lowest order, we do not use recurrence relations (see Section 2) for evaluating $I$. In [19] a method is described for computing $I$, using linear programming.

\section{Evaluating a Finite-Element Model Problem}

In [19] the bending thin-plate problem mentioned before, is evaluated. In this article that problem is considered with two differences with respect to [19]. In the first place the two cornerpoints joining the symmetry boundary and the free boundary, respectively, with the simply supported plate boundary, are double knots (in [19] extensions of $\Omega$ were used). In the second place, the number of elements covering $\Omega$ is considerably increased. This allows a first estimate of the rate of convergence as a function of element size, by comparing the numerical results with an accurate result from [17].

The upper half of the plate, partitioned into 48 triangles, is sketched in Fig. 4.1, together with the pulled knots. The same problem has been solved using 12 triangles (a change with a factor of 2 in the linear dimensions of the elements). The results are presented in Table 4.1. Considering the errors, the convergence behavior seems to be quadratic.

In [11] and [12] the same problem was treated using Heindl split-triangle quadratic elements. Here also a quadratic rate of convergence has been estimated.

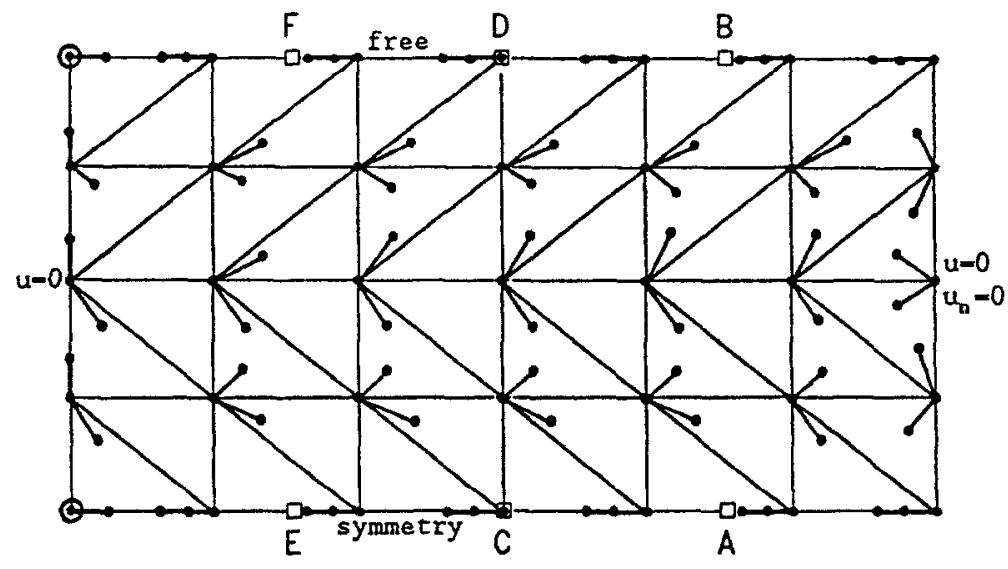

Fig. 4.1. Upper half of the bending plate. 
Table 4.1.

\begin{tabular}{llllll}
\hline & & & & \multicolumn{2}{c}{ Errors } \\
\cline { 5 - 6 } & & & & & \\
\cline { 4 - 6 } & $T=12$ & “Exact" & $T=48$ & $T=12$ \\
\hline$u_{\mathrm{A}}$ & 0.03781 & 0.03443 & 0.03860 & 0.0008 & 0.0042 \\
$u_{\mathrm{B}}$ & 0.03986 & 0.03549 & 0.04078 & 0.0009 & 0.0053 \\
$u_{\mathrm{C}}$ & 0.08112 & 0.07747 & 0.08241 & 0.0013 & 0.0049 \\
$u_{\mathrm{D}}$ & 0.08592 & 0.08080 & 0.08808 & 0.0022 & 0.0073 \\
$u_{\mathrm{E}}$ & 0.06854 & 0.06578 & 0.06955 & 0.0010 & 0.0038 \\
$u_{\mathrm{F}}$ & 0.07277 & 0.06934 & 0.07457 & 0.0018 & 0.0052 \\
\hline
\end{tabular}

\section{References}

1. C. DE BOOR, T. LYCHE, L. L. SCHUMAKER (1976): On calculating with B-splines, II. Integration. In: Numerical Methods of Approximation Theory (L. Collatz, G. Meinardus, H. Werner, eds.). Basel: Birkhäuser, pp. 123-146.

2. E. COHEN, T. LYCHE, R. F. RIESENFELD (1987): Cones and recurrence relations for simplex splines. Constr. Approx., 3:131-141.

3. W. DAHMEN (1979): Multivariate B-splines-recurrence relations and linear combinations of truncated powers. In: Multivariate Approximation Theory (W. Schempp, K. Zeller, eds.). Basel: Birkhäuser, pp. 64-82.

4. W. DAHMEN (1980): Konstruktion mehrdimensionaler B-splines und ihre Anwendungen auf Approximationsprobleme. In: Numerical Methods of Approximation Theory (L. Collatz, G. Meinardus, H. Werner, eds.). Basel: Birkhäuser, pp. 84-110.

5. W. DAHMEN, C. A. MiCChelli (1981): Computation of inner products of multivariate B-splines. Numer. Funct. Anal. Optim., 3:367-375.

6. W. DAhMEN, C. A. MiCChelli (1981): Numerical algorithms for least squares approximation by multivariate B-splines. In: Numerical Methods of Approximation Theory (L. Collatz, G. Meinardus, H. Werner, eds.). Basel: Birkhäuser, pp. 85-114.

7. W. DAHMEN, C. A. MICCHELLI (1982): On the linear independence of multivariate B-splines, I. Triangulation of simploids. SIAM J. Numer. Anal., 19:992-1012.

8. W. DAHMEN, C. A. MiCCHELli (1983): Recent progress in multivariate splines. In: Approximation Theory 4 (C. K. Chui, L. L. Schumaker, J. Ward, eds.). New York: Academic Press, pp. 27-121.

9. W. DAHMEN, C. A. MiCCHELLI (1983): Multivariate splines-a new constructive approach. In: Surfaces in CAGD (R. E. Barnhill, W. Boehm, eds.). Amsterdam: North-Holland, pp. 191-215.

10. R. H. J. Gmelig MeYling (1986): Polynomial Spline Approximation in Two Variables. Ph.D. Thesis, University of Amsterdam.

11. R. H. J. GMELIG MEYLING (1990): Numerical solution of the biharmonic equation using different types of bivariate spline functions. In: Algorithms for Approximation II (J. C. Mason, M. G. Cox, eds.). London: Chapman and Hall, pp. 369-376.

12. R. H. J. Gmelig MeYling (1988): Smooth Piecewise Polynomials as Conforming Finite Elements for Plate Bending Problems. Memorandum 747, Department of Applied Mathematics, University of Twente.

13. K. HOLLIG (1982): Multivariate splines. SIAM J. Numer. Anal., 19:1013-1031.

14. M. NEAMTU (1989): On the recurrence relation for multivariate B-splines with respect to dimension. Memorandum 786, Department of Applied Mathematics, University of Twente.

15. M. NEAMTU (1989): Multivariate divided differences and B-splines. In: Approximation Theory 6 (C. K. Chui, L. L. Schumaker, J. Ward, eds.). New York: Academic Press, pp. 445-448.

16. M. NeamTU (1990): Multivariate divided differences, I. Basic properties. Memorandum 875, Department of Applied Mathematics, University of Twente.

17. H. R. Schwarz (1988): Finite Element Methods. London: Academic Press. 
18. C. R. TRAAS (1986): Boundary conditions with bivariate quadratic B-splines. In: Approximation Theory 5 (C. K. Chui, L. L. Schumaker, J. Ward, eds.). New York: Academic Press, pp. 595-598.

19. C. R. TRAAS (1989): Approximation of surfaces constrained by a differential equation using simplex splines. In: Mathematical Methods of Computer Aided Geometric Design (T. Lyche, L. L. Schumaker, eds.). New York: Academic Press, pp. 593-599.

M. Neamtu

Department of Applied Mathematics

University of Twente

Enschede

The Netherlands
C. R. Traas

Department of Applied Mathematics

University of Twente

Enschede

The Netherlands 